\title{
Epilogue du PEK
}

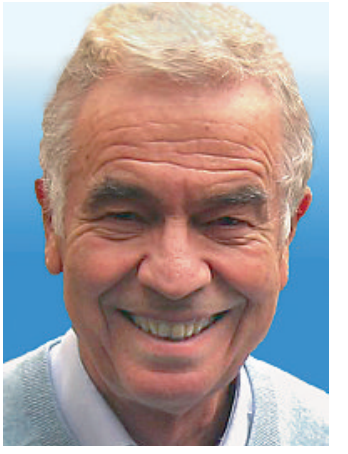

Hans Stalder

Le Prof. Stalder a été membre du review board international du PEK.
En 1998 le Département fédéral de l'Intérieur avait introduit provisoirement la médecine complémentaire (MC) pour 6 ans dans l'assurance de base et chargé l'Office Fédéral des Assurances Sociales (OFAS) de faire durant cette période une étude «PEK» (Projekt Evaluation Komplementärmedizin) afin d'évaluer efficacité, adéquation et économicité. Espérons que l'article «Ist ärztliche Komplementärmedizin wirtschaftlich?» paru dans le Bulletin du 5 mai 2010 sera le dernier sur cette étude, car la meilleure chose qui pourrait arriver au PEK serait de l'oublier rapidement, étant donné qu'il n'a guère répondu aux questions posées et n'a généré que de nouvelles controverses. La raison est que le PEK a été dès le début 1 . mal géré, 2 . mal exécuté et 3. mal interprété.

1. Mal géré: A la place de mettre au concours le projet de recherche et de le déléguer à des personnes compétentes, l'OFAS, non expert dans ce domaine, a gardé tout au long la supervision. Comme conséquence, le début des travaux scientifiques a été retardé de 4 ans, car à l'ignorance de l'OFAS s'est ajoutée une grande méfiance des médecins à participer à une étude

\section{La meilleure chose qui pourrait arriver au PEK serait de l'oublier rapidement}

dirigée par l'Etat. À cause de ses préjugés concernant la MC cette supervision a par ailleurs été contreproductive tout au long de l'étude. De plus, vers la fin de l'étude ceux-ci ont viré dans l'autre extrême sous la nouvelle direction. Sa décision de biffer la MC de l'assurance de base sans même laisser aboutir les études en cours et sans obtenir l'avis des experts internationaux du review board a provoqué un tollé dans les milieux de la MC et aboutit au lancement de l'initiative populaire.

2. Mal exécuté: L'OFAS, ayant décidé de faire une enquête comparative entre la MC et la médecine «classique» afin de déterminer l'économicité, a malheureusement choisi un groupe de recherche qui n'avait guère d'expérience dans les enquêtes auprès des médecins. Ainsi, malgré l'intervention des experts, les questionnaires adressés aux médecins et aux patients ont été mal conçus et trop compliqués, ce qui a contribué à rendre le retour encore plus faible: moins d'un tiers des Hôpitaux Universi de Genève. pour les médecins et de ce petit tiers seul $44 \%$ pour les patients (donc moins que $15 \%$ de la totalité possible). De plus, un avis négatif du comité d'éthique du canton de Berne a empêché une partie de l'étude à se réaliser ...

3. Mal interprété: Il est évident qu'un retour si faible rend toute comparaison entre la MC et la médecine classique aléatoire. Il se trouve que non seulement les médecins de la MC sont différents des autres - ils font plutôt une médecine de référence (comme les spécialistes) qu'une médecine de premier recours et leurs consultations sont plus longues -, mais aussi leurs patients: en MC il y a davantage de femmes, qui sont plus jeunes et qui se croient plus gravement et chroniquement malades, mais qui sont moins souvent hospitalisées et utilisent moins de médicaments. Selon votre préférence, ceci vous permet soit de conclure que la MC est plus coûteuse, car les consultations sont souvent de seconde intention et plus longues ou alors de dire qu'elle est plus avantageuse, car elle entraîne moins de coûts secondaires malgré le fait que les patientes se croient plus malades. Or, la seule conclusion honnête et valable du PEK est, que les deux médecines (par ailleurs souvent pratiquées par la même personne) s'adressent par des méthodes différentes à une clientèle différente pour des problèmes différents. De conclure par le PEK que l'une ou l'autre est meilleure ou économiquement avantageuse, et de donner même des chiffres, n'est pas seulement injustifié mais inadmissible d'un point de vue scientifique.

Le PEK n'a pas donné une réponse au problème autrement fascinant: Pourquoi la grande majorité de la population désire que la MC soit réintroduite dans l'assurance de base, malgré le fait qu'un effet supérieur au placébo ne puisse être démontré (à l'exception de la phytothérapie)? Car, ceci est aussi une conclusion du PEK: Par des méta-analyses l'Institut de Médecine Social et Préventive de Berne l'a encore une fois confirmé pour l'homéopathie et constaté que pour les autres branches il n'y a pas de données véritablement comparables.

Les discussions de nos parlementaires pour légiférer le vote populaire sur la MC ne se feront donc pas sur des bases scientifiques. Dommage pour les millions dépensés pour le PEK. Peut-être devraient-ils accepter les consultations en MC (plébiscité par le peuple), mais pas les médicaments (invalidés par la science)?

Hans Stalder* 